\title{
Application of Recurrence Quantification Analysis for Non-Linear Dynamical Systems
}

\author{
Himanshu Kumar shekhar, Chetan Kamble, Ashish Thakur, Sanjeet Kumar Bhagat
}

\begin{abstract}
The Recurrence plots (RPs) have been introduced in several different scientific and medical disciplines. The main purpose of recurrence plot is used to of identify the higher dimensional phase space trajectories. RPs are purely graphically representation which have been designed for the detection of hidden dynamical patterns and non-linearity present in the data, the evaluation of error which is caused by observational noise can be done by Recurrence Quantification Analysis (RQA). RQA method is initially used to minimize the error present in the given signals. RQA method is a basically a technique for the analysis of nonlinear data to quantify the number and duration of a dynamical systems. The recurrence plot is used for time series domain for multidimensional signal also. Recurrence is the property of non-stationary and dynamical system to characteristics the time series analysis in phase space trajectories. Recurrence Quantification Analysis is used to derive from recurrence plots, which are based upon distances matrices of time series.
\end{abstract}

Index Terms: Recurrence quantification analysis (RQA), Dynamical systems.

\section{INTRODUCTION}

Recurrence quantification analysis is the method for verification and analysis of the signal in time domain itself. The RQA method is not applicable for frequency domain to analysis the any physical signals. The recurrence plot is the method to draw the

Space trajectory for nonlinear time invariant and dynamics system. In this method we can analysis the nonlinear data with stationary systems. Recurrence is the property to revisit the points in the phase space trajectory. In signal processing application RQA method has been used. To compress the data and process through signal processing has been helped of RQA method. Many signals (sine waves) at a constant frequency will perfectly collapse to a fraction of one sine cycle with loss of no information. Recurrence plot. We can use as a form of square matrix. Another method also there to analysis or investigate the nonlinear systems such as the Lyapunov exponent, KolmogorovSinai entropy, correlation dimension, etc. but these tools are used for stationary but Recurrence plot can be used for nonstationary in time. For Non-linearand non-stationary system we can use RQA method. It can be identify the several Non- Linear systems. In the signal processing the noise play a very tough challenge. RQA method are used to identify the location of the events based on the phase space trajectory. So to express dynamic systems which are complex, we can use RQA. RQA method are used to identify the location of the events based on the phase space trajectory.

Revised Manuscript Received on December 15, 2019

Himanshu Kumar shekhar, Vignan's Foundation for Science, Technology and Research, Guntur, Andhra Pradesh

Chetan Kamble, Vignan's Foundation for Science, Technology and Research, Guntur, Andhra Pradesh

Ashish Thakur, Vignan's Foundation for Science, Technology and Research, Guntur, Andhra Pradesh

Sanjeet Kumar Bhagat, Vignan's Foundation for Science, Technology and Research, Guntur, Andhra Pradesh
Discharge in the presence of high electromagnetic interference. Recurrence plots (RPs) gives subjective information about dynamic system. To access some more subjective information we can use of RQA. RQA is based on the distributions of the diagonal, horizontal and vertical lines that are found in the RP. The tool which provides link between RPs and RQA which are widely use in fields such as physics, medicine, earth science and economics.

\section{LITERATURE REVIEW}

Recurrences tools are used for the first return maps, space time separation Plots return time and recurrence time statistics. However, more than a half century had to pass for recurrences to be comprehensively studied on numerical simulations and real measurements. The application of the recurrence matrix as a tool to visualize recurrences of higher-dimensional phase space trajectories. The main purpose of the recurrence plots and their quantification as a modern tool of nonlinear data analysis. In this thesis, basically talking about nonlinear dynamics of the systems, [1] In the chaos theory, there are two types of dynamics system first one is Differential equations and second one is difference equations. Differential equations derive the evolution of system in continuous time whereas difference equation derives the iterated maps in discrete time. [2] Recurrence analysis is a graphical method designed to locate hidden recurring patterns, non-stationary and structural changes, introduced in Eckmann et al. (1987, RQA determination index has been used.

The authors of [9] have proposed that the features of RQA can be with usual feature statistics to increase classification accuracy in the case of auditory scenes. Auditory scenes may involve a wide variety of sounds. By avoiding linearity and stationary assumptions, RQA features can complement traditional feature statistics and help dealing with the uncertainty. Given their simplicity, we foresee more applications in audio analysis tasks. Experiment results showed that, on certain time scales, the recurrence structures of abnormal heart rate fluctuation from subjects with congestive heart failure could be reflected more clearly by multi- scale RQA parameters. In [12], study examined the degree to which the quality and characteristics of students' essays could be modeled through dynamic natural language processing analyses. Undergraduate students $(\mathrm{n}=131)$ wrote timed, persuasive essays in response to an argumentative writing prompt. Recurrent patterns of the words in the essays were then analyzed using recurrence quantification analysis (RQA system under investigation.

The RQA analysis method is applicable for both one dimensional signal as well as the multi-dimensional signal. This method is used to analysis the signal behavior in only time domain approaches, the main disadvantage of the RQA method is, cannot visualize in frequency domain.

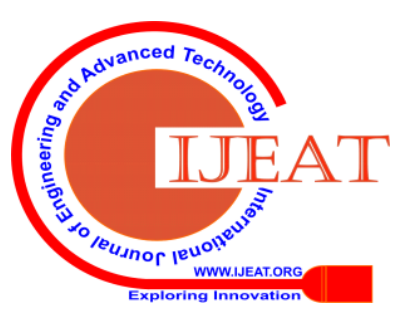




\section{Application of Recurrence Quantification Analysis for Non-Linear Dynamical Systems}

\section{RECURRENCE QUANTI-}

FICATION ANALYSIS DET $=$

$N$

$l=\operatorname{lmin}$

$N$

$i,=1$

l.p(l)

$R i, j$

Recurrence Quantification Analysis is the graphical measuring method that is used for the quantifies the recurrence plot. Firstly RPs method was used for the visualisation tool. It could not be used for the visualise the large data sets to overcome this problem the scientists Zbilutand Webber introduced the new method that is called the RQA (Recurrence Quantification Analysis). For quantifying the structure of RPs, RQA method is used. Recurrence point density and the diagonal and vertical line structures of the RP are the classified measures of the RQA. The technique can appraise the number and prolongation of dynamic system of being in nearly the same area in phase space

Where $\mathrm{P}$ (1) is the number of contingency of a diagonal of length. Most of the recurrent points form diagonal lines. Hence DET is close to 1 for fully deterministic signals. DET is close to 0 for random white noise because in RP, most of the recurrent points are single isolated points and form very few diagonal lines.

III(c) ENT (Entropy)

Entropy is defined as the complexity measure of the distribution of the diagonal lines in the recurrence plot (RP). Entropy is defined in term of average information carried by incoming sources .It is given by the following equation: trajectory is called Recurrence quantification analysis (RQA). It is a nonlinear data analysis. A short description

$$
\mathrm{ENT}=\sum_{l=l_{\min }}^{N} P(l) \cdot \log \mathrm{P}(l)
$$

of various measures of RQA is given below:

$$
\text { Where }(l)=\frac{P(l)}{\Gamma_{l=l_{\min }}^{N} P(l)}
$$

III (a) Recurrence Rate (RR)

The density of recurrent points in the RP plot is called as recurrence rate. This is excluded from the computation as there is always a diagonal on Line of Identity (LoI). This exclusion is given by the following equation:

$$
\begin{gathered}
\mathrm{RR}=\frac{1}{2}{ }^{N} R \quad \text {, where } \mathrm{R} \text { is } \\
N^{2} \quad i, \quad i j
\end{gathered}
$$

It refers to the Shannon entropy w.r.t the probability of finding a diagonal line of exactly length 1 . The value is small for periodic signals or uncorrelated noise, and is high for chaotic systems. The valuable information about

Structure of the RP can be obtained

By the computation of the measures based on the diagonal lines and their The recurrence matrix.

Where $\mathrm{R}$ is the recurrence matrix and $\mathrm{N}$ is length of the data. The constraint is the probability that the state/measurement will repeat. This limiting case can be used for an infinitely long signal.

III (b) Determinism (DET)
Determinism (DET) is the ratio of recurrence points that form a diagonal structure, where $(l)$ is the histogram of the length of diagonal line. The percentage of
recurrent
points is
quantified
by

Determinism (DET). This form a diagonal of minimum length

lmin. By default lminis taken as 2. DET is defined as:

III (d) DIV (Divergence)

Divergence in recurrence plot is defined the reciprocal of the longest diagonal lines. So in chaotic systems divergence is directly dependent on the maximum Lyapunov exponent.

\section{RESULTS AND DISCUSSION}

1) The following composite sinusoidal signal as shown below in the Fig.4.1 has been taken as an input to obtain the recurrence plot :

$(t)=\cos t+\sin (t)+\sin (2 t)$

2

wher, $t=0$ to 150 (In seconds)

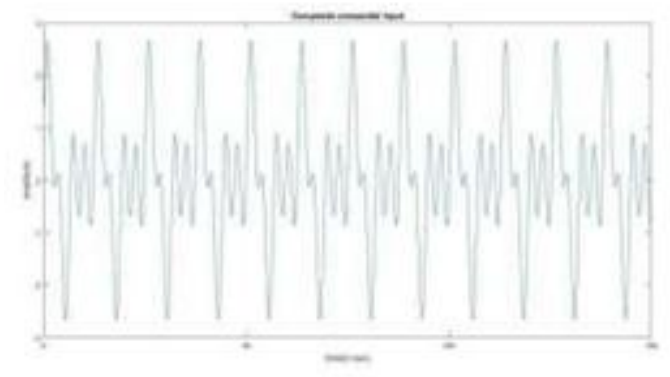

Fig 4.1 Input composite sinusoidal signal As shown below in the Fig.4.2, we have got the recurrence plot for the input signal taken as shown in the Fig.4.1.

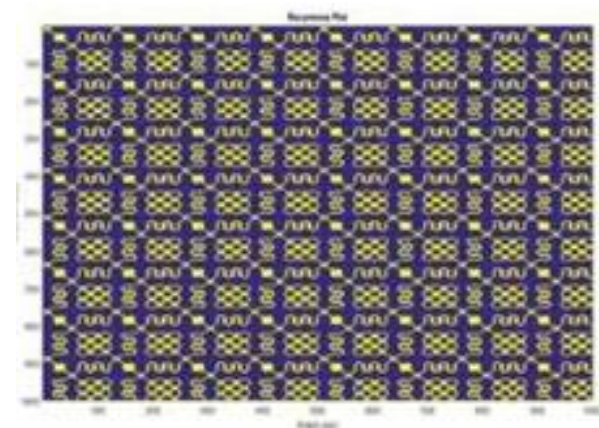

Fig 4.2 Recurrence plot of composite signal

The output as shown in the Fig. 4.2 represents the periodic or oscillating behavior of the recurrence plot of the input taken. Here, diagonal lines represent the recurrence plot of the composite sinusoidal signal. The frequency ratio (number of diagonal lines dividing main diagonal line) has come out as zero because the output has no diagonal line intersecting the main diagonal line.

2) The following sinc with noise signal as shown below in the Fig.4.3 has been taken as an input to obtain the recurrence plot:

$(t)=s i(4 t)+$ noise

noise $=1 / 3 \times r a(11001)$ 
where, 'randn'is some

random noise function

The sinc function has been compressed with 4 times the variable 'time'.

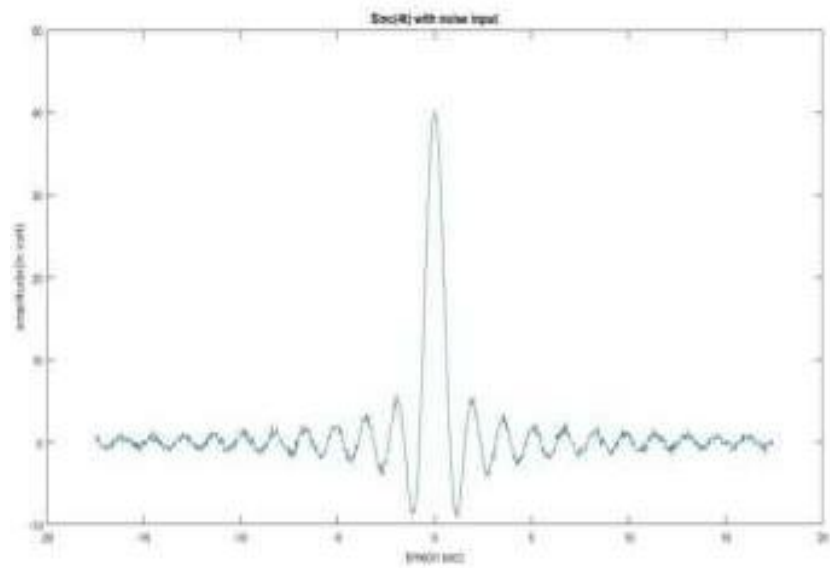

Fig 4.3 Input compressed sinc signal with noise As shown below in the Fig.4.3

we have got the recurrence plot for the input signal taken as shown in the Fig.4.4.

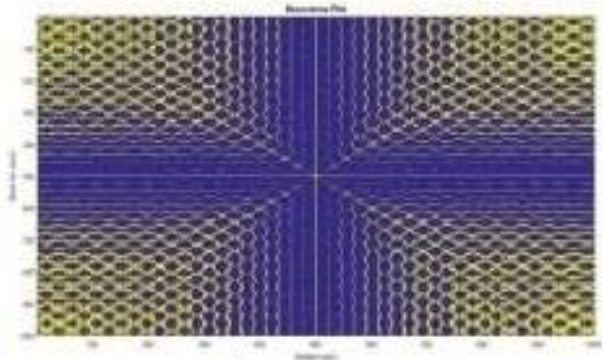

Fig 4.4 Recurrence plot of compressed sinc signal with noise

The output as shown in the Fig. 4.4 represents the both oscillating and stationary behavior of the recurrence plot of the input taken. The frequency ratio (number of diagonal lines dividing main diagonal line) has come out as 1 because the output has single diagonal line crossing the main diagonal line. The Fig.4.6 represents that the system taken as

Input for the recurrence plot is both stationary and oscillating in nature. The liminality of the plot is very high. If value of recurrence point in vertical line and

Horizontal line increase then liminality decreases. The single point of intersection represents that the determinant is approximately 0 .

\section{RESULT TABLE:}

\begin{tabular}{|l|c|l|c|c|}
\hline Time series signal & $\begin{array}{c}\text { Determinism } \\
\text { (DET) }\end{array}$ & $\begin{array}{l}\text { Recurrence } \\
\text { Rate } \\
\text { (RR) }\end{array}$ & $\begin{array}{c}\text { Entropy } \\
\text { (ENT) }\end{array}$ & $\begin{array}{c}\text { Divergence } \\
\text { (DIV) }\end{array}$ \\
\hline $\begin{array}{l}\text { 1)Compressed } \\
\text { composite } \\
\text { signal }\end{array}$ & $\begin{array}{c}4.789 \times \\
10^{-4}\end{array}$ & 0.2653 & 0.1640 & $9.90 \times$ \\
$10^{-5}$ & & & $7.89 \times$ \\
\hline $\begin{array}{l}\text { 2) Unit step } \\
\text { signal with } \\
\text { noise }\end{array}$ & $\begin{array}{c}8.1907 \times \\
10^{-6}\end{array}$ & 0.7318 & 0.0274 & $10^{-5}$ \\
\hline $\begin{array}{l}\text { 3) Compressed } \\
\text { sinc signal } \\
\text { withnoise }\end{array}$ & $2.35 \times$ & 0.2122 & 0.0164 & $9.05 \times$ \\
\hline
\end{tabular}

Time series signal

Determinism

(DET) Recurrence Rate

(RR) Entropy

(ENT) Divergence

(DIV)

1) Compressed $4.789 \times \quad 0.2653 \quad 0.1640 \quad 9.90 \times$ composite signal $10-4$

$10-5$

2) Unit step $\quad 8.1907 \times 0.7318 \quad 0.0274 \quad 7.89 \times$

signal with noise $10-6 \quad 10-5$

3) Compressed 2.35× $0.2122 \quad 0.0164 \quad 9.05 \times$

sinc signal with noise $10-4$

As shown in the Table 4.1, parameters for different signals have been calculated and tabulated in the Table 4.1 as shown above. It represents that the value of determinant is very low for the stationary signal and high for the periodic or oscillating signals. Also, the recurrence rate is high for the composite oscillating signal and stationary

Signal, but low as compared to the other signals. The value of entropy is high for the oscillating signal as compared to stationary signal and low for compressed oscillating signals with phase. The value of divergence is almost equivalent as compared to all the signals except the Gaussian

\section{CONCLUSION}

The application of Recurrence Quantification Analysis is investigated. DET and RR are the two parameters of RQA which are calculated for the different time series signal using MATLAB. The

Signal with more DET and RR is considered as the less noise free signal. Here Gaussian noise and sine with phase has a high DET and compressed composite signal with noise has high RR value.

\section{REFERENCES}

1. S. Strogatz, Nonlinear Dynamics and Chaos, Perseus Books, 2000

2. J. P. Eckmann, S. O. Kamphorst, and D. Ruelle, "Recurrence plots of Dynamical systems," Europhys. Lett. vol. 4, pp. 973-977,1987.

3. Chiara Mocenni*, Angelo Facchini, Antonio Vicino, "Comparison of recurrence quantification methods for the analysis of temporal and spatial chaos",2010.

4. Marco Thiel, M Carmen Romano, JurgenKurths, R. Meucci, E. Allaria, F. T. Arecchi, "Influence of observational noise on the recurrence quantification analysis”, Physica. D,Nonlinear Phenomena,2002.

5. H. Kantz, T. Schreiber, Nonlinear Time Series Analysis (University Press, Cambridge, 1997.

6. Pratyasa Bhui and Nilanjan Senroy, "Application of Recurrence Quantification Analysis to Power System Dynamic Studies", IEEE TRANSACTIONS ON POWER SYSTEMS, VOL. 31,NO. 1, January2016.

7. Norbert Marwan and Jurgen Kurths, "Cross Recurrence Plots and Their Application", Nova Science Publisher, Inc.,2004.

8. Charles L. Webber, Jr and Joseph

9. P. Zbilut, "Recurrence Quantification Analysis of Non- linear Dynamical System", 1992.

10. George Tzagkaraki and Thomas Dionysopoulos, "Recurrence quantification analysis of denoised index returns via alpha-stable modeling of wavelet coefficients: Detecting switching volatility regimes", Studies in Nonlinear Dynamics and Economics,2015.

11. ChengyuHuo, Yi Lu and Xiaolin Huang, Hongxing Liu, Xinbao Ning "Multi- scale Recurrence Quantification Analysis of Heartbeat Interval Series in Healthy vs. Heart Failure Subjects", 7th International Conference on BioMedical Engineering and Informatics, 2014. 